\title{
ON THE WATER-BAG MODEL OF DISPERSIONLESS KP HIERARCHY
}

\author{
JEN-HSU CHANG \\ DEPARTMENT OF COMPUTER SCIENCE \\ NATIONAL DEFENSE UNIVERSITY \\ TAUYUAN, TAIWAN \\ E-MAIL: JHCHANG@CCIT.EDU.TW
}

\begin{abstract}
We investigate the bi-Hamiltonian structure of the waterbag model of dKP for two component case. One can establish the third-order and first-order Hamiltonian operator associated with the waterbag model. Also, the dispersive corrections are discussed.

Key Words: waterbag model, bi-Hamiltonian structure, recursion operator, symmetry constraints, dispersive corrections

MSC (2000): 35Q58, 37K10, 37K 35
\end{abstract}

\section{INTRODUCTION}

The dispersionless KP hierarchy(dKP or Benney moment chain) is defined by

$$
\partial_{t_{n}} \lambda(p)=\left\{\lambda(p), B_{n}(p)\right\}, \quad n=1,2, \cdots .
$$

where the Lax operator $\lambda(p)$ is

$$
\lambda(p)=p+\sum_{1}^{\infty} v_{n+1} p^{-n}
$$

and it can be used to define a set of polynomials:

$$
B_{n}(p)=\frac{\left[\lambda^{n}(p)\right]_{+}}{n}, \quad i=1,2,3, \cdots \quad t_{1}=x .
$$

Here $[\cdots]_{+}$denotes a non-negative part of the Laurent series $\lambda^{n}(p)$. For example,

$$
B_{2}=\frac{p^{2}}{2}+v_{2}, \quad B_{3}=\frac{p^{3}}{3}+v_{2} p+v_{3} .
$$

Moreover, the bracket in (11) stands for the natural Poisson bracket on the space of functions of the two variables $(x, p)$ :

$$
\{f(x, p), g(x, p)\}=\partial_{x} f \partial_{p} g-\partial_{x} g \partial_{p} f .
$$


The compatibility of (11) will imply the zero-curvature equation

$$
\partial_{m} B_{n}(p)-\partial_{n} B_{m}(p)=\left\{B_{n}(p), B_{m}(p)\right\} .
$$

If we denote $t_{2}=y$ and $t_{3}=t$, then the equation (44) for $m=2, n=3$ gives

$$
\begin{aligned}
& v_{3 x}=v_{2 y} \\
& v_{3 y}=v_{2 t}-v_{2} v_{2 x},
\end{aligned}
$$

from which the dKP equation is $\operatorname{derived}\left(v_{2}=v\right)$

$$
v_{y y}=\left(v_{t}-v v_{x}\right)_{x} .
$$

According to the dKP theory [1, 18, 22, 37, there exists a wave function $S\left(\lambda, x, t_{2}, t_{3}, \cdots\right)$ such that $p=S_{x}$ and satisfies the HamiltonianJacobian equation

$$
\frac{\partial S}{\partial t_{n}}=\left.B_{n}(p)\right|_{p=S_{x}} .
$$

It can be seen that the compatibility of (6) also implies the zerocurvature equation (44). Now, we expand $B_{n}(p)$ as

$$
B_{n}(p(\lambda))=\frac{\left[\lambda^{n}(p)\right]_{+}}{n}=\frac{\lambda^{n}}{n}-\sum_{i=0}^{\infty} G_{i n} \lambda^{-i-1},
$$

where the coefficients can be calculated by the residue form:

$$
G_{i n}=-r e s_{\lambda=\infty}\left(\lambda^{i} B_{n}(p) d \lambda\right)=\frac{1}{i+1} \operatorname{res}_{p=\infty}\left(\lambda^{i+1} \frac{\partial B_{n}(p)}{\partial p} d p\right),
$$

which also shows the symmetry property

$$
G_{i n}=G_{n i} .
$$

Moreover, from

$$
\frac{\partial B_{m}(\lambda)}{\partial t_{n}}=\frac{\partial B_{n}(\lambda)}{\partial t_{m}}
$$

this implies the integrability of $G_{i n}$ as expressed in terms of the free energy $\mathcal{F}$ (dispersionless $\tau$ function) [37]

$$
G_{i n}=\frac{\partial^{2} \mathcal{F}}{\partial t_{i} \partial t_{n}} .
$$

For example, the series inverse to (22) is

$$
p=\lambda-\frac{\mathcal{F}_{11}}{\lambda}-\frac{\mathcal{F}_{12}}{2 \lambda^{2}}-\frac{\mathcal{F}_{13}}{3 \lambda^{3}}-\frac{\mathcal{F}_{14}}{4 \lambda^{4}}-\cdots,
$$

where $\mathcal{F}_{1 n}$ are polynomials of $v_{2}, v_{3}, \cdots, v_{n+1}$ and in fact

$$
h_{n} \equiv \frac{\mathcal{F}_{1 n}}{n}=\operatorname{res}_{p=\infty} \frac{\lambda^{n}}{n} d p
$$


are the conserved densities for the dKP hierarchy (11). In [5] 6], it's proved that dKP hierarchy (11) is equivalent to the dispersionless Hirota equation

$$
D(\lambda) S\left(\lambda^{\prime}\right)=-\log \frac{p(\lambda)-p\left(\lambda^{\prime}\right)}{\lambda},
$$

where $D(\lambda)$ is the operator $\sum_{n=1}^{\infty} \frac{1}{n \lambda^{n}} \frac{\partial}{\partial t_{n}}$.

Next, we consider the symmetry constraint [3, 4

$$
\mathcal{F}_{x}=\sum_{i=1}^{n} c_{i}\left(S_{i}-\tilde{S}_{i}\right)
$$

where $S_{i}=S\left(\lambda_{i}\right)$ and $\tilde{S}_{i}=S\left(\tilde{\lambda}_{i}\right), \lambda_{i}, \tilde{\lambda}_{i}$ are some sets of points, and $c_{i}$ are arbitrary constants. Notice that from (7) we know

$$
D(\lambda) \mathcal{F}_{x}=\lambda-p .
$$

On the other hand, by (8) and (9), we also have

$$
\begin{aligned}
D(\lambda) \mathcal{F}_{x} & =D(\lambda) \sum_{i=1}^{N} c_{i}\left(S_{i}-\tilde{S}_{i}\right)=\sum_{i=1}^{N} c_{i}\left(D(\lambda) S_{i}-D(\lambda) \tilde{S}_{i}\right) \\
& =-\sum_{i=1}^{N} c_{i} \log \frac{p-p_{i}}{p-\tilde{p}_{i}},
\end{aligned}
$$

where $p=p(\lambda), p_{i}=p\left(\lambda_{i}\right)$ and $\tilde{p}_{i}=p\left(\tilde{\lambda}_{i}\right)$.

Hence we obtain the non-algebraic reduction("waterbag" model) 3 , 16] of dKP hierarchy

$$
\lambda=p-\sum_{i=1}^{N} c_{i} \log \frac{p-p_{i}}{p-\tilde{p}_{i}}=p+\sum_{s=1}^{\infty} v_{s+1} p^{-s}
$$

where

$$
v_{s+1}=\sum_{i=1}^{N} \frac{c_{i}\left(p_{i}^{s}-\tilde{p}_{i}^{s}\right)}{s} .
$$

One remarks that in the limit $\tilde{\lambda}_{i}=\lambda_{i}+\epsilon_{i}, \epsilon_{i} \rightarrow 0$, keeping $c_{i} \epsilon_{i}=$ $d_{i}$ to be constant, the Sato function (10) reproduces the Zakharov's reduction 38

$$
\lambda=p+\frac{d_{1}}{p-\tilde{p}_{1}}+\frac{d_{2}}{p-\tilde{p}_{2}}+\cdots+\frac{d_{N}}{p-\tilde{p}_{N}},
$$


which is the algebraic reduction of dKP hierarchy.

From (10), we have

$$
B_{2}(p)=\frac{1}{2} p^{2}+\sum_{i=1}^{N} c_{i}\left(p_{i}-\tilde{p}_{i}\right) .
$$

So $\left(t_{2}=y\right)$

$$
\partial_{y} \lambda=\left\{\lambda, \sum_{i=1}^{N} c_{i}\left(p_{i}-\tilde{p}_{i}\right)\right\}
$$

This will imply

$$
\begin{aligned}
& \partial_{y} p_{\lambda}=\partial_{x}\left[\frac{1}{2} p_{\lambda}^{2}+\sum_{i=1}^{N} c_{i}\left(p_{i}-\tilde{p}_{i}\right)\right] \\
& \partial_{y} \tilde{p}_{\lambda}=\partial_{x}\left[\frac{1}{2} \tilde{p}_{\lambda}^{2}+\sum_{i=1}^{N} c_{i}\left(p_{i}-\tilde{p}_{i}\right)\right] .
\end{aligned}
$$

For simplicity, in this paper we only consider the case $N=1$, i.e.,

$$
\begin{aligned}
& \partial_{y} p_{1}=\partial_{x}\left[\frac{1}{2} p_{1}^{2}+c_{1}\left(p_{1}-\tilde{p}_{1}\right)\right] \\
& \partial_{y} \tilde{p}_{1}=\partial_{x}\left[\frac{1}{2} \tilde{p}_{1}^{2}+c_{1}\left(p_{1}-\tilde{p}_{1}\right)\right],
\end{aligned}
$$

and the Lax operator (10) is truncated to become

$$
\lambda=p-c_{1} \log \frac{p-p_{1}}{p-\tilde{p}_{1}} .
$$

The equation (14) can also be written as the Hamiltonian system

$$
\left[\begin{array}{c}
p_{1 y} \\
\tilde{p}_{1 y}
\end{array}\right]=\left[\begin{array}{cc}
\frac{1}{c_{1}} & 0 \\
0 & -\frac{1}{c_{1}}
\end{array}\right] \partial_{x}\left[\begin{array}{c}
\frac{\delta H_{3}}{\delta p_{1}} \\
\frac{\delta H_{3}}{\delta \tilde{p}_{1}}
\end{array}\right],
$$

where $\delta$ is the variation derivative and

$$
H_{3}=\frac{1}{3} \int d x\left[c_{1}\left(p_{1}^{3}-\tilde{p}_{1}^{3}\right)+3 c_{1}^{2}\left(p_{1}-\tilde{p}_{1}\right)^{2}\right]
$$

A bi-Hamiltonian structure is defined as (for the case of dKP)

$$
\left[\begin{array}{c}
p_{1 y} \\
\tilde{p}_{1 y}
\end{array}\right]=\mathbb{J}_{1}\left[\begin{array}{c}
\frac{\delta H_{3}}{\delta p_{1}} \\
\frac{\delta H_{3}}{\delta \tilde{p}_{1}}
\end{array}\right]=\mathbb{J}_{2}\left[\begin{array}{c}
\frac{\delta H}{\delta p_{1}} \\
\frac{\delta H}{\delta \tilde{p}_{1}}
\end{array}\right],
$$


where

$$
\mathbb{J}_{1}=\left[\begin{array}{cc}
\frac{1}{c_{1}} & 0 \\
0 & -\frac{1}{c_{1}}
\end{array}\right] \partial_{x}
$$

and $H$ is some Hamiltonian such that $\mathbb{J}_{2}$ is also a Hamiltonian operator (Jacobi identity), which is compatible with $\mathbb{J}_{1}$, i.e., $\mathbb{J}_{1}+c \mathbb{J}_{2}$ is also a Hamiltonian one for any complex number $c$ [12, 13, 29, 23. We hope to find $\mathbb{J}_{2}$ and the related Hamiltonian $H$.

Besides, from (11) and (15) we know that $v_{2}=v=p_{1}-\tilde{p}_{1}$. Then by the theory of symmetry constraints of KP [8, 20, 21, 35, hierarchy one can consider the dispersive corrections for the waterbag model (15).

This paper is organized as follows. In the next section, we construct the third-order bi-Hamiltonian structure. Section 3 is devoted to establishing first-order bi-Hamiotonian structure using WDVV equation in topological field theory. In section 4, we discuss the dispersive corrections. In the final section, one discusses some problems to be investigated.

\section{Third-Order Bi-Hamiltonian Structure}

In this section, we investigate the bi-Hamiltonian structure of the two component case (14). To find the bi-Hamiltonian structure, one can introduce the coordinates

$$
u=p_{1}+\tilde{p}_{1}, \quad v=p_{1}-\tilde{p}_{1}
$$

to rewrite equation (14) as

$$
\begin{aligned}
\left(\begin{array}{l}
u \\
v
\end{array}\right)_{y} & =\left(\begin{array}{c}
\frac{u^{2}+v^{2}}{4}+2 c_{1} v \\
\frac{u v}{2}
\end{array}\right)_{x} \\
& =\frac{1}{c_{1}}\left(\begin{array}{cc}
0 & D_{x} \\
D_{x} & 0
\end{array}\right)\left(\begin{array}{c}
\frac{\delta H_{3}}{\delta u} \\
\frac{\delta H_{3}}{\delta v}
\end{array}\right),
\end{aligned}
$$

where

$$
\begin{aligned}
H_{3} & =\frac{1}{3} \int d x\left[c_{1}\left(p_{1}^{3}-\tilde{p}_{1}^{3}\right)+3 c_{1}^{2}\left(p_{1}-\tilde{p}_{1}\right)^{2}\right] \\
& =\frac{1}{3} \int d x\left[c_{1} \frac{3 u^{2} v+v^{3}}{4}+3 c_{1}^{2} v^{2}\right]=\int d x h_{3}
\end{aligned}
$$

and $\delta$ is the variational derivative. One can observe the conserved density

$$
h_{3}=\frac{1}{3}\left[c_{1} \frac{3 u^{2} v+v^{3}}{4}+3 c_{1}^{2} v^{2}\right]
$$


has the separable property

$$
\frac{\partial^{2} h_{3}}{\partial u^{2}} / \frac{\partial^{2} h_{3}}{\partial v^{2}}=\frac{c_{1} v}{2} /\left(\frac{c_{1} v}{2}+2 c_{1}^{2}\right)=\frac{1}{\mu(v)},
$$

where

$$
\mu(v)=\frac{v+4 c_{1}}{v}=1+\frac{4 c_{1}}{v} .
$$

Hence we can identify the equation (16) as the generalized gas dynamic Hamiltonian system or separable Hamiltonian system [32, 34. Therefore, according to the separable Hamiltonian theory in [2, 32, we know that the third-order Hamiltonian operator

$$
J_{2}=D_{x} U_{x}^{-1} D_{x} U_{x}^{-1} \sigma D_{x},
$$

where

or

$$
U_{x}=\left(\begin{array}{cc}
u_{x} & \mu(v) v_{x} \\
v_{x} & u_{x}
\end{array}\right) \quad \text { and } \quad \sigma=\left(\begin{array}{cc}
0 & 1 \\
1 & 0
\end{array}\right)
$$

$$
U_{x}^{-1}=\frac{1}{u_{x}^{2}-\mu(v) v_{x}^{2}}\left(\begin{array}{cc}
u_{x} & -\mu(v) v_{x} \\
-v_{x} & u_{x}
\end{array}\right),
$$

is compatible with the first order Hamiltonian operator

$$
J_{1}=\left(\begin{array}{cc}
0 & D_{x} \\
D_{x} & 0
\end{array}\right)=\sigma D_{x} .
$$

So we can write the equation (16) as the bi-Hamiltonian structure

$$
\begin{aligned}
\left(\begin{array}{l}
u \\
v
\end{array}\right)_{y} & =\frac{1}{c_{1}} J_{1}\left(\begin{array}{c}
\frac{\delta H_{3}}{\delta u} \\
\frac{\delta H_{3}}{\delta v}
\end{array}\right)=\frac{1}{c_{1}} J_{2}\left(\begin{array}{c}
\frac{\delta H_{5}}{3 \delta u} \\
\frac{\delta H_{5}}{3 \delta v}
\end{array}\right) \\
& =\frac{1}{c_{1}} D_{x}\left(\begin{array}{c}
\frac{h_{5}, u u v}{3} \\
\frac{h_{5}, v v u}{3}
\end{array}\right),
\end{aligned}
$$

where

$$
\begin{aligned}
H_{5} & =\int h_{5} d x=\frac{1}{5} \int \operatorname{res}_{p=\infty}\left(\lambda^{5} d p\right) d x \\
& =\frac{1}{5} \int\left\{c_{1}\left(p_{1}^{5}-\tilde{p}_{1}^{5}\right)+10 c_{1}^{3}\left(p_{1}-\tilde{p}_{1}\right)^{3}+\frac{20}{3} c_{1}^{2}\left(p_{1}-\tilde{p}_{1}\right)\left(p_{1}^{3}-\tilde{p}_{1}^{3}\right)\right. \\
& \left.+\frac{5}{2} c_{1}^{2}\left(p_{1}^{2}-\tilde{p}_{1}^{2}\right)^{2}\right\} d x \\
& =\frac{1}{5} \int\left\{\frac{c_{1}}{16}\left(5 u^{4} v+10 u^{2} v^{3}+v^{5}\right)+10 c_{1}^{3} v^{3}+\frac{15}{2} c_{1}^{2} u^{2} v^{2}\right. \\
& \left.+\frac{5}{3} c_{1}^{2} v^{4}\right\} d x .
\end{aligned}
$$


Next, one will want to find all the conserved density $F(u, v)$ of equation (16). It's not difficult to see that if $\int F(u, v) d x$ is a conserved quantity if and only if that $F(u, v)$ satisfies the wave equation

$$
F_{u u}=\frac{F_{v v}}{\mu(v)}
$$

The wave equation (17) leads to two fundamental hierarchies of conserved densities 32, 34

$$
\begin{aligned}
& F_{N}=\sum_{n=0}^{\left[\frac{N}{2}\right]} \frac{u^{N-2 n}}{(N-2 n) !}\left(\partial_{v}^{-2} \mu(v)\right)^{n} \cdot v \\
& \tilde{F}_{N}=\sum_{n=0}^{\left[\frac{N+1}{2}\right]} \frac{u^{N+1-2 n}}{(N+1-2 n) !}\left(\partial_{v}^{-2} \mu(v)\right)^{n} \cdot 1 .
\end{aligned}
$$

Here $\partial_{v}^{-1}=\int_{0}^{v} d v$ and $\partial_{v}^{-1}$ acts on all the factors standing to the right of it. For example,

$$
\left(\partial_{v}^{-2} \mu(v)\right)^{2} \cdot v=\int_{0}^{v} d v \int_{0}^{v}\left[\mu(v) \int_{0}^{v} d v \int_{0}^{v} v \mu(v) d v\right] d v .
$$

For reference, we list the first few members of each sequence in the generalized gas dynamic system (16): $\left(\mu(v)=1+\frac{4 c_{1}}{v}\right)$

$$
\begin{aligned}
& F_{0}=v, \quad F_{1}=u v \\
& F_{2}=\frac{1}{2} u^{2} v+\frac{1}{6} v^{3}+2 c_{1} v^{2} \\
& F_{3}=\frac{1}{6} u^{3} v+u\left(\frac{1}{6} v^{3}+2 c_{1} v^{2}\right) \\
& F_{4}=\frac{1}{24} u^{4} v+\frac{u^{2}}{2}\left(\frac{1}{6} v^{3}+2 c_{1} v^{2}\right)+\left(\frac{1}{120} v^{5}+\frac{2}{9} c_{1} v^{4}+\frac{4}{3} c_{1}^{2} v^{3}\right)
\end{aligned}
$$

and

$$
\begin{aligned}
\tilde{F}_{0} & =u, \quad \tilde{F}_{1}=\frac{1}{2} u^{2}+\frac{1}{2} v^{2}+4 c_{1}(v \ln v-v) \\
\tilde{F}_{2} & =\frac{1}{6} u^{3}+u\left[\frac{1}{2} v^{2}+4 c_{1}(v \ln v-v)\right] \\
\tilde{F}_{3} & =\frac{1}{24} u^{4}+\frac{1}{2} u^{2}\left[\frac{1}{2} v^{2}+4 c_{1}(v \ln v-v)\right]+\frac{1}{24} v^{4}+\frac{2}{3} c_{1} v^{3} \ln v-\frac{8}{9} c_{1} v^{3} \\
& +8 c_{1}^{2} v^{2} \ln v-20 c_{1}^{2} v^{2} \\
\tilde{F}_{4} & =\frac{1}{120} u^{5}+\frac{1}{6} u^{3}\left[\frac{1}{2} v^{2}+4 c_{1}(v \ln v-v)\right]+u\left[\frac{1}{24} v^{4}+\frac{2}{3} c_{1} v^{3} \ln v-\frac{8}{9} c_{1} v^{3}\right. \\
& \left.+8 c_{1}^{2} v^{2} \ln v-20 c_{1}^{2} v^{2}\right]
\end{aligned}
$$


In fact, one can see that

$$
F_{N-1}=\frac{2^{N-1}}{c_{1}(N-1) !} h_{N}=\frac{2^{N-1}}{c_{1} N !} r e s_{p=\infty}\left(\lambda^{N} d p\right), \quad N \geq 1
$$

and from (18) we also have (for $N \geq 1$ )

$$
\begin{aligned}
& \frac{\partial F_{N}}{\partial u}=F_{N-1} \\
& \frac{\partial \tilde{F}_{N}}{\partial u}=\tilde{F}_{N-1}
\end{aligned}
$$

Moreover, we notice that the recursion operator

$$
\hat{R}=J_{2} J_{1}^{-1}=D_{x} U_{x}^{-1} D_{x} U_{x}^{-1}
$$

is the square of a simpler first-order recursion operator

$$
R=D_{x} U_{x}^{-1} .
$$

Then we can easily check that, using (17) and (20),

$$
\begin{aligned}
& R^{-1} \sigma D\left(\begin{array}{c}
\frac{\partial F_{N}}{\partial u} \\
\frac{\partial F_{N}}{\partial v}
\end{array}\right)=R^{-1}\left(\begin{array}{c}
\frac{\partial F_{N}}{\partial v} \\
\frac{\partial F_{N}}{\partial u}
\end{array}\right)_{x}=U_{x}\left(\begin{array}{c}
\frac{\partial F_{N}}{\partial v} \\
\frac{\partial F_{N}}{\partial u}
\end{array}\right) \\
& =\left(\begin{array}{c}
\frac{\partial F_{N+1}}{\partial v} \\
\frac{\partial F_{N+1}}{\partial u}
\end{array}\right)_{x}=\sigma D\left(\begin{array}{c}
\frac{\partial F_{N+1}}{\partial u} \\
\frac{\partial F_{N+1}}{\partial v}
\end{array}\right),
\end{aligned}
$$

and similarly for $\tilde{F}_{N}$. Hence the dKP hierarchy of (16) can be obtained using the recursion operator, i.e.,

$$
\left(\begin{array}{l}
u \\
v
\end{array}\right)_{t_{n}}=\left(R^{-1}\right)^{n-2} \frac{\sigma}{c_{1}} D\left(\begin{array}{c}
\frac{\partial h_{3}}{\partial u} \\
\frac{\partial h_{3}}{\partial v}
\end{array}\right), n \geq 2 .
$$

However, the Lax representation of hierarchy generated by $\tilde{F}_{N}$ is not found.

Also, using the recursion operator (21), one can construct a hierarchy of higher order Hamiltonian densities $\hat{F}_{m}, m=1,2,3, \cdots$, with $m$ indicating the order of derivatives on which they depend, and the corresponding commuting bi-Hamiltonian system [31]:

$$
\left(\begin{array}{l}
u \\
v
\end{array}\right)_{\tau_{m}}=\hat{R}^{m}\left(\begin{array}{l}
1 \\
0
\end{array}\right)=J_{1}\left(\begin{array}{c}
\frac{\delta \hat{F}_{m}}{\delta u} \\
\frac{\delta \hat{F}_{m}}{\delta v}
\end{array}\right)=J_{2}\left(\begin{array}{c}
\frac{\delta \hat{F}_{m-1}}{\delta u} \\
\frac{\delta \hat{F}_{m-1}}{\delta v}
\end{array}\right),
$$


where

$$
\begin{aligned}
& \hat{F}_{0}=\int x v d x \\
& \hat{F}_{1}=-\frac{1}{2} \int \frac{v_{x}}{u_{x}^{2}-\mu(v) v_{x}^{2}} d x=-\frac{1}{2} \int \frac{v_{x}}{u_{x}^{2}-\left(1+\frac{4 c_{1}}{v}\right) v_{x}^{2}} d x .
\end{aligned}
$$

All the flows of (22) will commute with the generalized gas dynamic system (16). For example, for $n=1$, we have, after a simple calculation,

$$
\begin{aligned}
\left(\begin{array}{l}
u \\
v
\end{array}\right)_{\tau_{1}} & =\hat{R}\left(\begin{array}{l}
1 \\
0
\end{array}\right) \\
& =\left(\begin{array}{c}
\frac{\mu^{2} v_{x}^{3} v_{x x}+3 \mu u_{x}^{2} v_{x} v_{x x}+\mu \mu^{\prime} v_{x}^{5}+\mu^{\prime} u_{x}^{2} v_{x}^{3}-u_{x}^{3} u_{x x}-3 \mu v_{x}^{2} u_{x} u_{x x}}{\left(u_{x}^{2}-\mu v_{x}^{2}\right)^{3}} \\
\frac{\mu v_{x}^{3} u_{x x}+3 u_{x}^{2} v_{x} u_{x x}-v_{x x} u_{x}^{3}-3 \mu v_{x}^{2} u_{x} v_{x x}-2 \mu^{\prime} u_{x} v_{x}^{4}}{\left(u_{x}^{2}-\mu v_{x}^{2}\right)^{3}}
\end{array}\right)_{x},
\end{aligned}
$$

where

$$
\mu^{\prime}=\frac{d \mu}{d v}=-\frac{4 c_{1}}{v^{2}} .
$$

Finally, one remarks that there exists a Lagrangian local in the velocity fields for the equation (16) ( up to a scaling):

$$
\mathcal{L}=\frac{v_{x} u_{t}-u_{x} v_{t}}{u_{x}^{2}-\mu v_{x}^{2}}-2 v .
$$

The local Lagrangian will exist in bi-Hamiltonian structure with a pair of first and third order Hamiltonian operators [30, 32.

\section{Free Energy and Bi-Hamiltonian Structure}

In this section, we investigate the relations between bi-Hamiltonian structure and free energy. Then the compatible first-order Hamiltonian operators can be constructed.

Now, we want to find the free energy associated with the dKP hierarchy (11) for the Lax operator of the form (15). Suppose we are given two first-order Hamiltonian operators $\hat{J}_{1}$ and $\hat{J}_{2}\left(\partial=\partial_{x}\right)$

$$
\begin{aligned}
\hat{J}_{1} & =\left(\begin{array}{ll}
0 & 1 \\
1 & 0
\end{array}\right) \partial \stackrel{\text { def }}{=} \eta_{1}^{i j} \partial \\
\hat{J}_{2} & =\left(\begin{array}{ll}
g^{11}(t) & g^{12}(t) \\
g^{21}(t) & g^{22}(t)
\end{array}\right) \partial+\left(\begin{array}{cc}
\Gamma_{1}^{11}(t) & \Gamma_{1}^{12}(t) \\
\Gamma_{1}^{21}(t) & \Gamma_{1}^{22}(t)
\end{array}\right) t_{x}^{1}+\left(\begin{array}{cc}
\Gamma_{2}^{11}(t) & \Gamma_{2}^{12}(t) \\
\Gamma_{2}^{21}(t) & \Gamma_{2}^{22}(t)
\end{array}\right) t_{x}^{2} \\
& \stackrel{\text { def }}{=} g^{i j}(t) \partial+\Gamma_{k}^{i j}(t) t_{x}^{k} .
\end{aligned}
$$


They are both Poisson brackets of hydrodynamic type introduced by Dubrovin and Novikov 10, 11. The bi-Hamiltonian structure means that $\hat{J}_{1}$ and $\hat{J}_{2}$ have to be compatible, i.e, $\hat{J}=\hat{J}_{1}+\alpha \hat{J}_{2}$ must also be a Hamiltonian structure for all value of $\alpha$. This compatibility condition implies that, for any $\alpha$, the metric is referred as flat pencil. The geometric setting in which to understand flat pencil ( or biHamiltonian structure of hydrodynamic system) is the Frobenius manifold [12, 13, 14, 15. One way to define such manifolds is to construct a function $\mathbb{F}\left(t^{1}, t^{2}, \cdots, t^{m}\right)$ such that the associated functions,

$$
c_{i j k}=\frac{\partial^{3} \mathbb{F}}{\partial t^{i} \partial t^{j} \partial t^{k}}
$$

satisfy the following conditions.

- The matrix $\eta_{i j}=c_{1 i j}$ is constant and non-degenerate. This together with the inverse matrix $\eta^{i j}$ are used to raise and lower indices. On such a manifold one may interpret $\eta_{i j}$ as a flat metric.

- The functions $c_{j k}^{i}=\eta^{i r} c_{r j k}$ define an associative commutative algebra with a unity element. This defines a Frobenius algebra on each tangent space $T^{t} \mathcal{M}$.

Equations of associativity give a system of non-linear PDE for $\mathbb{F}(t)$

$$
\frac{\partial^{3} \mathbb{F}(t)}{\partial t^{\alpha} \partial t^{\beta} \partial t^{\lambda}} \eta^{\lambda \mu} \frac{\partial^{3} \mathbb{F}(t)}{\partial t^{\mu} \partial t^{\gamma} \partial t^{\sigma}}=\frac{\partial^{3} \mathbb{F}(t)}{\partial t^{\alpha} \partial t^{\gamma} \partial t^{\lambda}} \eta^{\lambda \mu} \frac{\partial^{3} \mathbb{F}(t)}{\partial t^{\mu} \partial t^{\beta} \partial t^{\sigma}}
$$

These equations constitute the Witten-Dijkgraaf-Verlinde-Verlinde (or WDVV) equations. On such a manifold one may introduce a second flat metric defined by

$$
g^{i j}=\partial^{i} \partial^{j} \mathbb{F}+\partial^{j} \partial^{i} \mathbb{F},
$$

where

$$
\partial^{i}=\eta^{i \varsigma} \partial_{t^{\varsigma}}
$$

and the contravariant Levi-Civita connection is

$$
\Gamma_{k}^{i j}=\partial^{i} \partial^{j} \partial_{t^{k}} \mathbb{F},
$$

This metric, together with the original metric $\eta^{i j}$, define a flat pencil (i.e, $\eta^{i j}+\alpha g^{i j}$ is flat for any value of $\alpha$ ). Thus, one automatically obtains a bi-Hamiltonian structure from a Frobenius manifold $\mathcal{M}$. The corresponding Hamiltonian densities are defined recursively by the formula

$$
\frac{\partial^{2} \psi_{\alpha}^{(n)}}{\partial t^{i} \partial t^{j}}=c_{i j}^{k} \frac{\partial \psi_{\alpha}^{(n-1)}}{\partial t^{k}}
$$


where $n \geq 1, \alpha=1,2, \cdots, m$, and $\psi_{\alpha}^{0}=\eta_{\alpha \epsilon} t^{\epsilon}$. The integrability conditions for this systems are automatically satisfied when the $c_{i j}^{k}$ are defined as above.

For the waterbag hierarchy (11) (15), it is obvious that

$$
t^{1}=u=\psi_{2}^{(o)}, t^{2}=v=\psi_{1}^{(0)}
$$

and those $c_{i j}^{k}$ can be determined by (25)

$$
\frac{\partial^{2} \psi_{1}^{(n)}}{\partial t^{i} \partial t^{j}}=c_{i j}^{k} \frac{\partial \psi_{1}^{(n-1)}}{\partial t^{k}}
$$

where, using (19),

$$
\psi_{1}^{(n)}=F_{n}=\frac{2^{n}}{c_{1}(n+1) !} r e s_{p=\infty}\left(\lambda^{n+1} d p\right), \quad n \geq 0 .
$$

Simple calculations can get

$$
\begin{aligned}
& c_{11}^{1}=1, \quad c_{12}^{1}=c_{21}^{1}=0, \quad c_{22}^{1}=1+\frac{4 c_{1}}{t^{2}}=\mu(v), \\
& c_{11}^{2}=c_{22}^{2}=0, \quad c_{21}^{2}=c_{12}^{2}=1,
\end{aligned}
$$

By (26), we can get immediately free energy

$$
\begin{aligned}
\mathbb{F}\left(t^{1}, t^{2}\right) & =\frac{1}{2}\left(t^{1}\right)^{2} t^{2}+2 c_{1}\left(t^{2}\right)^{2} \log t^{2}+\frac{1}{6}\left(t^{2}\right)^{3} \\
& + \text { quadratic terms }
\end{aligned}
$$

We notice that the free energy (27) has no quasi-homogeneity condition and, however, the free energy associated with the Benney hierarchy is quasi-homogeneous [7].

After choosing suitable quadratic terms, then from the free energy (27), using (23) and (24), one can construct $\hat{J}_{2}$ as follows:

$$
\begin{aligned}
\hat{J}_{2} & =\left(\begin{array}{ll}
2 t^{2} & 2 t^{1} \\
2 t^{1} & 2 t^{2}
\end{array}\right) \partial+\left(\begin{array}{ll}
0 & 1 \\
1 & 0
\end{array}\right) t_{x}^{1}+\left(\begin{array}{ll}
1 & 0 \\
0 & 1
\end{array}\right) t_{x}^{2} \\
& =\left(\begin{array}{ll}
t^{2} \partial_{x}+\partial_{x} t^{2} & t^{1} \partial_{x}+\partial_{x} t^{1} \\
t^{1} \partial_{x}+\partial_{x} t^{1} & t^{2} \partial_{x}+\partial_{x} t^{2}
\end{array}\right) .
\end{aligned}
$$

Here we remove the non-analytic part of $g^{i j}$, i.e., $\ln t^{2}$. Also, one can verify directly that $\hat{J}_{2}$ is a Hamiltonian operator and is compatible with $\hat{J}_{1}$. We notice that the constant $c_{1}$ won't appear in $\hat{J}_{2}$.

Now, using the recursion operator

$$
\hat{\mathbb{R}}=\hat{J}_{2} \hat{J}_{1}^{-1}=\left(\begin{array}{cc}
t^{1}+\partial_{x} t^{1} \partial_{x}^{-1} & t^{2}+\partial_{x} t^{2} \partial_{x}^{-1} \\
t^{2}+\partial_{x} t^{2} \partial_{x}^{-1} & t^{1}+\partial_{x} t^{1} \partial_{x}^{-1}
\end{array}\right),
$$


one can construct a hierarchy by

$$
\left(\begin{array}{c}
t^{1} \\
t^{2}
\end{array}\right)_{\tilde{\tau}_{m}}=\hat{\mathbb{R}}^{m}\left(\begin{array}{c}
\frac{t^{1}}{6} \\
\frac{t^{2}}{6}
\end{array}\right)_{x}, \quad m \geq 1
$$

For example, for $m=1$ and $m=2$, a simple calculation can yield

$$
\begin{aligned}
& \left(\begin{array}{c}
t^{1} \\
t^{2}
\end{array}\right)_{\tilde{\tau}_{1}}=\hat{\mathbb{R}}\left(\begin{array}{c}
\frac{t^{1}}{6} \\
\frac{t^{2}}{6}
\end{array}\right)_{x}=\left(\begin{array}{c}
\frac{1}{4}\left[\left(t^{1}\right)^{2}+\left(t^{2}\right)^{2}\right] \\
\frac{1}{2} t^{1} t^{2}
\end{array}\right)_{x}, \\
& \left(\begin{array}{c}
t^{1} \\
t^{2}
\end{array}\right)_{\tilde{\tau}_{2}}=\hat{\mathbb{R}}^{2}\left(\begin{array}{c}
\frac{t^{1}}{6} \\
\frac{t^{2}}{6}
\end{array}\right)_{x}=5\left(\begin{array}{c}
\frac{1}{12}\left(t^{1}\right)^{3}+\frac{1}{4} t^{1}\left(t^{2}\right)^{2} \\
\frac{1}{12}\left(t^{2}\right)^{3}+\frac{1}{4} t^{2}\left(t^{1}\right)^{2}
\end{array}\right)_{x},
\end{aligned}
$$

which are slightly different from the $y$ flow (16) and $t_{3}($ or $t)$ flow of the dKP hierarchy (11), respectively:

$$
\begin{aligned}
\left(\begin{array}{c}
t^{1} \\
t^{2}
\end{array}\right)_{t} & =\frac{2}{3 c_{1}} J_{1}\left(\begin{array}{c}
\frac{\delta H_{4}}{\delta t^{1}} \\
\frac{\delta H_{4}}{\delta t^{2}}
\end{array}\right) \\
& =\left(\begin{array}{c}
\frac{1}{12}\left(t^{1}\right)^{3}+\frac{1}{4} t^{1}\left(t^{2}\right)^{2}+2 c_{1} t^{1} t^{2} \\
\frac{1}{12}\left(t^{2}\right)^{3}+\frac{1}{4} t^{2}\left(t^{1}\right)^{2}+c_{1}\left(t^{2}\right)^{2}
\end{array}\right)_{x},
\end{aligned}
$$

where

$$
H_{4}=\int h_{4} d x=\frac{1}{4} \int\left\{c_{1}\left[\frac{\left(t^{1}\right)^{3} t^{2}+\left(t^{2}\right)^{3} t^{1}}{2}\right]+6 c_{1}^{2} t^{1}\left(t^{2}\right)^{2}\right\} d x
$$

By comparisons between them, one can see that the non-homogeneous terms (or higher- order $c_{1}$ terms) of the water-bag hierarchy could be removed in the hierarchy (28). In this way, one can say that the hierarchy (28) is perturbed, up to some scalings, by the water-bag hierarchy with a perturbation parameter $c_{1}$.

Remark:According to the Kodama-Gibbons formulation [18, the Riemann invariants $\lambda_{1}, \lambda_{2}$ of (29) are given by,

$$
\begin{aligned}
\lambda_{1} & =\lambda\left(u_{1}\right)=\frac{t^{1}+\sqrt{\left(t^{2}\right)^{2}+4 c_{1} t^{2}}}{2}-c_{1} \ln \frac{\sqrt{\left(t^{2}\right)^{2}+4 c_{1} t^{2}}-t^{2}}{\sqrt{\left(t^{2}\right)^{2}+4 c_{1} t^{2}}+t^{2}} \\
(30)_{2} & =\lambda\left(u_{2}\right)=\frac{t^{1}-\sqrt{\left(t^{2}\right)^{2}+4 c_{1} t^{2}}}{2}+c_{1} \ln \frac{\sqrt{\left(t^{2}\right)^{2}+4 c_{1} t^{2}}-t^{2}}{\sqrt{\left(t^{2}\right)^{2}+4 c_{1} t^{2}}+t^{2}},
\end{aligned}
$$

where $u_{1}$ and $u_{2}$ are the real roots of $\left.\frac{d \lambda}{d p}\right|_{p=u_{1}, u_{2}}=0$, i.e.,

$$
u_{1}=\frac{t^{1}+\sqrt{\left(t^{2}\right)^{2}+4 c_{1} t^{2}}}{2}, \quad u_{2}=\frac{t^{1}-\sqrt{\left(t^{2}\right)^{2}+4 c_{1} t^{2}}}{2},
$$


and the characteristic speeds are

$$
\hat{v}_{1}=\left.\frac{d \Omega_{3}(p)}{d p}\right|_{p=u_{1}}, \quad \hat{v}_{2}=\left.\frac{d \Omega_{3}(p)}{d p}\right|_{p=u_{2}} .
$$

Then the equation (29) can be written as

$$
\left(\begin{array}{l}
\lambda_{1} \\
\lambda_{2}
\end{array}\right)_{t}=\left(\begin{array}{cc}
\hat{v}_{1} & 0 \\
0 & \hat{v}_{2}
\end{array}\right)\left(\begin{array}{l}
\lambda_{1} \\
\lambda_{2}
\end{array}\right)_{x} .
$$

Also, a simple calculation shows that, using (30), the flat metric $(d s)^{2}=$ $d t^{1} d t^{2}$ becomes, in Riemann's invariants,

$$
(d s)^{2}=\eta_{11}(t)\left(d \lambda_{1}\right)^{2}+\eta_{22}(t)\left(d \lambda_{2}\right)^{2},
$$

where

$$
\begin{aligned}
& \eta_{11}(t)=\operatorname{res}_{p_{1}} \frac{(d p)^{2}}{d \lambda}=\frac{1}{\left.\frac{d^{2} \lambda}{d p^{2}}\right|_{p=u_{1}}}=\frac{t^{2}}{\sqrt{\left(t^{2}\right)^{2}+4 t^{2} c_{1}}} \\
& \eta_{22}(t)=\operatorname{res}_{p_{2}} \frac{(d p)^{2}}{d \lambda}=\frac{1}{\left.\frac{d^{2} \lambda}{d p^{2}}\right|_{p=u_{2}}}=-\frac{t^{2}}{\sqrt{\left(t^{2}\right)^{2}+4 t^{2} c_{1}}} .
\end{aligned}
$$

Since it's known that waterbag reduction (15) is not scaling invariant [16], we can verify that the metric

$$
\begin{aligned}
(d \tilde{s})^{2} & =\frac{\eta_{11}}{u_{1}}\left(d \lambda_{1}\right)^{2}+\frac{\eta_{22}}{u_{2}}\left(d \lambda_{2}\right)^{2} \\
& =\frac{t^{2}}{4}\left(d t^{1}\right)^{2}+\frac{t^{1}}{2} d t^{1} d t^{2}+\left(\frac{t^{2}}{4}+c_{1}\right)\left(d t^{2}\right)^{2}
\end{aligned}
$$

is no more flat 15. Hence from the theory of Darboux-Egrov metric 12 , one believes that there is no first-order bi-Hamiltonian structure for (29) (or (16)). However, we know that (31) is (semi-)Hamiltonian [16, 33] and it probably will have a compatible non-local Poisson brackets of hydrodynamic type [24, 25, 26, 27, 28, deserving further investigations.

Finally, one notices that the metric (32) can also be obtained using the free energy (27)

$$
\mathbb{F}\left(t^{1}, t^{2}\right)=\frac{1}{2}\left(t^{1}\right)^{2} t^{2}+2 c_{1}\left(t^{2}\right)^{2} \log t^{2}+\frac{1}{6}\left(t^{2}\right)^{3}+2 c_{1}\left(t^{1}\right)^{2} .
$$

\section{DISPERSIVE CORRECTIONS}

In this section, one will investigate the dispersive corrections of watebag model from the theory of symmetry constraints of KP hierarchy. 
Let's briefly describe the KP hierarchy [9] The Lax operator of KP hierarchy is

$$
L=\partial_{X}+\sum_{n=1}^{\infty} V_{n+1} \partial_{X}^{-n}
$$

and the KP hierarchy is determined by the Lax equation $\left(\partial_{n}=\frac{\partial}{\partial T_{n}}, T_{1}=\right.$ $X)$

$$
\partial_{n} L=\left[B_{n}, L\right]
$$

where $B_{n}=\frac{1}{n} L_{+}^{n}$ is the differential part of $L^{n}$. For example $\left(T_{2}=\right.$ $\left.Y, T_{3}=T\right)$

$$
\begin{aligned}
V_{2 Y} & =\frac{V_{2 X X}}{2}+V_{3 X} \\
V_{3 Y} & =\frac{1}{2} V_{3 X X}+V_{4 X}+V_{2} V_{2 X} \\
V_{2 T} & =\frac{1}{3} V_{2 X X X}+V_{3 X X}+V_{4 X}+2 V_{2} V_{2 X} .
\end{aligned}
$$

Eliminating $V_{3}$ and $V_{4}$, we can obtain the $\mathrm{KP}$ equation $\left(V_{2}=V\right)$

$$
V_{T}=\frac{1}{4} V_{X X X}+V V_{X}+\partial_{X}^{-1} V_{Y Y}
$$

which also can be described as the compatibility condition for the eigenfunction $\phi$

$$
\begin{aligned}
\phi_{Y} & =\left(\frac{1}{2} \partial_{X}^{2}+V\right) \phi \\
\phi_{T} & =\left(\frac{1}{3} \partial_{X}^{3}+V \partial+V_{3}+V_{X}\right) \phi .
\end{aligned}
$$

To get dKP equation (5), one simply takes $T_{n} \rightarrow \varepsilon T_{n}=t_{n}$ in the KP equation (36), with

$$
\partial_{T_{n}} \rightarrow \varepsilon \partial_{t_{n}} \quad \text { and } \quad V\left(T_{n}\right) \rightarrow v\left(t_{n}\right),
$$

to obtain the $\mathrm{dKP}$ equation when $\varepsilon \rightarrow 0$. Thus the dispersive term $\frac{1}{4} V_{X X X}$ is removed. Moreover, letting

$$
\phi=\exp \frac{S}{\varepsilon}
$$

in (37), we also have the equation (6) for $n=2,3$

$$
\begin{aligned}
S_{y} & =\frac{1}{2} S_{x}^{2}+v_{2} \\
S_{t} & =\frac{1}{3} S_{x}^{3}+v_{2} S_{x}+v_{3}
\end{aligned}
$$

when $\varepsilon \rightarrow 0$. The compatibility $S_{t y}=S_{y t}$ will yield the dKP equation (51). 
Since $v=p_{1}-\tilde{p}_{1}$, from the theory of symmetry constraints of KP hierarchy [20, 21], one can assume the constraint

$$
V=\left(\ln \phi_{1}-\ln \phi_{2}\right)_{X}
$$

where $\phi_{1}$ and $\phi_{2}$ are arbitrary eigenfunctions, i.e., they both satisfy equations (37). We remark that if $\phi_{1}=\exp \frac{S_{1}}{\varepsilon}, \quad \phi_{2}=\exp \frac{S_{2}}{\varepsilon}$ and $X \rightarrow \varepsilon X=x$, then $V(X, Y, T) \rightarrow v(x, y, t)=p_{1}-\tilde{p}_{1}$, where

$$
p_{1}=S_{1 x}, \quad \tilde{p}_{1}=p_{2}=S_{2 x}
$$

when $\varepsilon \rightarrow 0$.

Then equations (37) become, $i=1,2$,

$$
\begin{aligned}
\phi_{i y} & =\frac{1}{2} \phi_{i X X}+\left(\ln \phi_{1}-\ln \phi_{2}\right)_{X} \phi_{i} \\
\phi_{i T} & =\frac{1}{3} \phi_{i X X X}+\left(\ln \phi_{1}-\ln \phi_{2}\right)_{X} \phi_{i X} \\
& +\left[V_{3}+\left(\ln \phi_{1}-\ln \phi_{2}\right)_{X X}\right] \phi_{i} .
\end{aligned}
$$

By equations (33) and (39), a simple calculation yields

$$
V_{3}=\frac{\left[\left(\ln \phi_{1}\right)_{X}\right]^{2}-\left[\left(\ln \phi_{2}\right)_{X}\right]^{2}}{2}
$$

and by equations (34) and (39), one obtains

$$
V_{4}=\frac{1}{3}\left\{\left[\left(\ln \phi_{1}\right)_{X}\right]^{3}-\left[\left(\ln \phi_{2}\right)_{X}\right]^{3}\right\}
$$

One notices that the dispersionless limits of (41) and (42) are $v_{3}=\frac{p_{1}^{2}-\tilde{p}_{1}^{2}}{2}$ and $v_{4}=\frac{p_{1}^{3}-\tilde{p}_{1}^{3}}{3}$, respectively. Unfortunately, using (35) and (40), after a tedious calculation, one has

$$
\left[\left(\ln \phi_{1}\right)_{X X}\right]^{2}-\left[\left(\ln \phi_{2}\right)_{X X}\right]^{2}=0,
$$

which is a contradiction since $\phi_{1}$ and $\phi_{2}$ are arbitrary eigenfunctions. This means that (40) is not a higher-order Lie-Backlund symmetry of (39) or the constraint (38) is not admissable 20, 35.

Also, we can consider the following case generalizing (38)

$$
V=\left[1-f\left(\partial_{X}\right)\right]^{-1}\left[\ln \phi_{1}-\ln \phi_{2}\right]_{X}
$$

or

$$
V-f\left(\partial_{X}\right) V=\left[\ln \phi_{1}-\ln \phi_{2}\right]_{X}
$$

where

$$
f\left(\partial_{X}\right)=a_{1} \partial_{X}+a_{2} \partial_{X}^{2}+\cdots+a_{n} \partial_{X}^{n}, \quad a_{i} \text { being constants. }
$$


The dispersionless limit of (43) is also $v=p_{1}-\tilde{p}_{1}$. Similarly, from (33) and (34) and (39), one can get

$$
\begin{aligned}
V_{3} & =\frac{1}{2}\left[1-f\left(\partial_{X}\right)\right]^{-1}\left\{\left[\left(\ln \phi_{1}\right)_{X}\right]^{2}-\left[\left(\ln \phi_{2}\right)_{X}\right]^{2}\right\} \\
V_{4} & =\frac{1}{3}\left[1-f\left(\partial_{X}\right)\right]^{-1}\left\{\left[\left(\ln \phi_{1}\right)_{X}\right]^{3}-\left[\left(\ln \phi_{2}\right)_{X}\right]^{3}\right. \\
& \left.+3\left[f\left(\partial_{X}\right) V^{2}\right]-3 V\left[f\left(\partial_{X}\right) V\right]\right\} .
\end{aligned}
$$

Both the dispersionless limits of $V_{3}$ and $V_{4}$ are $v_{3}=\frac{p_{1}^{2}-\tilde{p}_{1}^{2}}{2}$ and $v_{4}=$ $\frac{p_{1}^{3}-\tilde{p}_{1}^{3}}{3}$.

Now, using (35) and (40), a lengthy calculation shows that

$$
2 V\left[f\left(\partial_{X}\right) V_{X}\right]-\left[f\left(\partial_{X}\right)\left(V^{2}\right)_{X}\right]=\left[\left(\ln \phi_{1}\right)_{X X}\right]^{2}-\left[\left(\ln \phi_{2}\right)_{X X}\right]^{2},
$$

which is also an contradiction. Hence in the general case (43), it's also not admissible. For example, letting $f\left(\partial_{X}\right)=\partial_{X}$, we have $\left(V_{2}=V\right)$

$$
\begin{aligned}
V & =\left(1-\partial_{x}\right)^{-1}\left[\ln \phi_{1}-\ln \phi_{2}\right]_{X} \\
V_{3} & =\frac{1}{2}\left[1-\partial_{X}\right]^{-1}\left\{\left[\left(\ln \phi_{1}\right)_{X}\right]^{2}-\left[\left(\ln \phi_{2}\right)_{X}\right]^{2}\right\} \\
V_{4} & =\frac{1}{3}\left[1-\partial_{X}\right]^{-1}\left\{\left[\left(\ln \phi_{1}\right)_{X}\right]^{3}-\left[\left(\ln \phi_{2}\right)_{X}\right]^{3}+3 V_{X}\right\}
\end{aligned}
$$

and (44) becomes

$$
-2 V_{X}^{2}=\left[\left(\ln \phi_{1}\right)_{X X}\right]^{2}-\left[\left(\ln \phi_{2}\right)_{X X}\right]^{2}
$$

or, using (45),

$$
\left(\ln \phi_{1}-\ln \phi_{2}\right)_{X X}= \pm\left(1-\partial_{X}\right) \sqrt{\frac{\left[\left(\ln \phi_{2}\right)_{X X}\right]^{2}-\left[\left(\ln \phi_{1}\right)_{X X}\right]^{2}}{2}},
$$

which puts constraint on $\phi_{1}$ and $\phi_{2}$. Therefore (45) is not admissible.

\section{CONCLUDING REMARKS}

We have investigated the bi-Hamiltonian structure and dispersive corrections of the waterbag model for two component. After introducing suitable coordinates, one can identify (16) as a separable Hamiltonian system and thus a third-order bi-Hamiltonian structure is obtained. Also, using the recursion relation of conserved densities, we can find the free energy associated with water-bag model in WDVV equation of the topological field theory and thus establish the firstorder bi-Hamiltonian structure. However,the hierarchy constructed by recursion operator is not the same as dKP hierarchy. Also, one considers the dispersive corrections from the theory of symmetric constraints of KP theory. Some calculations show that these dispersive corrections 
are not admissible. Finally, one remarks that the solutions of the waterbag model can be found using the hodograph method in [18, 19.

Several questions remain to be overcome. Firstly, from the theory of non-local Poisson brackets of hydrodynamic type [26, 28, one believes the bi-Hamiltonian structure of (29) (or (16)) deserves further investigations, especially that the free energy (27) is not quasi-homogeneous. Secondly, as we see in section 4, the integrable dispersive corrections of the waterbag model are still unknown. The main difficulty is in the quantization of the Lax operator (10), i.e., $p \rightarrow \partial_{X}$. The exact form is not clear and needs further investigations [36]. Ultimately, we hope to generalize the results in section 2 to the general case, for example, fourcomponent case. But the computation is more involved. One hopes to address these questions elsewhere.

\section{Acknowledgments}

The author is grateful to Professor Maxim V.Pavlov for his stimulating discussions on bi-Hamiltonian theory. He also thanks Professor I.A.B. Strachan for valuable suggestions. He thanks the National Science Council for support under grant no. NSC 94-2115-M-014-001. 


\section{REFERENCES}

[1] Aoyama S and Kodama Y, Topological Landau-Ginzburg theory with a rational potential and the dKP hierarchy,Comm. Math. Phys., 182(1996), p.185-220

[2] M.Arik, F.Neyzi, Y.Nutku, P.J. Olver, J.Verosky, Multi-Hamiltonian structure of the Born-Infeld equation, J. Math. Phys., 30(1989), p.1338-p.1344

[3] L.V. Bogdanov and B.G. Konopelchenko, Symmetry constrains for dispersionless integrable equations and systems of hydrodynamic type, Phys. Lett. A, 330(2004), p.448-p.459, arXiv:nlin.SI/0312013 2003

[4] L.V. Bogdanov and B.G. Konopelchenko, On dispersionless BKP hierarchy and its reductions, arXiv:nlin.SI/0411046, 2004

[5] A.Boyarsky, A.Marshakov, O.Ruchayckiy, P.Wiegmann, A. Zabrodin, On Associativity equation in dispersionless integrable hierarchies, Phys. Lett. B, 515(2001),p.483-p.492

[6] R. Carroll and Y. Kodama, Solution of the dispersionless Hirota equations, J. Phys. A, 28(1995), p.6373-6387

[7] Jen-Hsu Chang and Ming-Hsien Tu, On the Benney Hierarchy: free energy, string equation and quantization, J.Phys. A: Math. Gen. 34(2001), p.251-p.272

[8] Yi Cheng, Constraints of the Kadomtsev-Petviashvili hierarchy, J. Math. Phys., Vol.33, No.11(1992), p.3774-p.3782

[9] L.A. Dickey, Soliton Equations and Hamiltonian Systems,Second Edition, World Scientific, 2003

[10] B.Dubrovin and S.P. Novikov, Hydrodynamics of weakly deformed soliton lattices, differential geometry and Hamiltonian theory, Russ. Math. Surv., 44(1989), p.35-124

[11] B.A. Dubrovin and S.P. Novikov, Hamiltonian formalism of one-dimensional systems of hydrodynamic type and the Bogolyubov-Whitham averaging method, Soviet Math. Dokl., 27(1983), p.665-p.669

[12] B.Dubrovin, Geometry of 2D topological field theories in "Integrable systems and Quantum Groups", Springer Lecture Notes in Math., V 1620, 1996, p.120348

[13] B.Dubrovin, Flat pencils of metrics and Frobenius manifolds, math.DG/9803106

[14] B.Dubrovin and Y.J.Zhang, Normal Forms of Hierarchies of Integrable PDES, Frobenius Manifolds and Gromov-Witten Invariants, arXiv: math.DG/0108160, 2001

[15] B.Dubrovin, Integrable systems in Topological Field Theory, Nucl. Phys. B379(1992), 627-689

[16] J.Gibbons and S.P. Tsarev, Reductions of the Benney Eequations, Phys. Lett. A, 211(1996), p.19-p.24

[17] J.Gibbons and S.P. Tsarev, Conformal maps and reductions of the Benney equations, Phys. Lett. A, 258(1999), p.263-p.271

[18] Y. Kodama and J. Gibbons, Integrability of dispersionless KP hierarchy, Proceedinds Fourth Workshop on Nonlinear and Turbulent Process in Physics, World Scientific, 1990, p.166-180

[19] Y.Kodoma, A solution method for dKP Equation, Prog. Theor. Phys. Supplement, No.94, 1988, p.184-p.194 
[20] B. Konopelchenko and W. Strampp, Reductions of (2+1)-dimensional integrable systems via mixed potential-eigenfunction constraints, J. Phys. A: Math. and Gen., Vol(33), No. 11(1992), p.4399-p.4411

[21] B. Konopelchenko and W. Strampp, New reductions of Kadomtsev-Petviashvili and two-dimensional Toda lattice hierarchies via symmetry constraints, J. Math. Phys., Vol. 33, No. 11(1992), p.3676-p.3686

[22] I.Krichever, The $\tau$-function of the universal Whitham hierarchy, matrix models and Topological Field Theories, Comm. Pure Appl. Math., 47(1994), p.437-475

[23] F.Magri, A simple model of the integrable Hamiltonian equation, J.Math. Phys., 19(1978), p.1156-p.1162

[24] A.Ya. Maltsev, On the compatible weakly non-local Poisson brackets of hydrodynamic type, Intern. Jour. of Math. Math. Sci., 32(2002) ,p.587-p.614

[25] A.Ya. Maltsev and S.P. Novikov, On the local systems Hamiltonian in the weakly non-local Poisson brackets, Physica D, 156(2001), p.53-p.80

[26] I.O.Mokhov, Compatible nonlocal Poisson brackets of hydrodynamic type, and integrable hierarchies related to them, arXiv:math.DG/0201242

[27] I.O.Mokhov, Quasi-Frobenius algebras and their integrable N-parametric deformations generated by compatible $(\mathrm{N}$ x N)-metrics of constant Riemannian curvature, Theor.Math.Phys. 136 (2003), 908-916

[28] Nonlocal Hamiltonian operators of hydrodynamic type with flat metrics, integrable hierarchies and the equations of associativity, arXiv:math.DG/0406292

[29] Luen-Chau Li, Classical r-Matrix and compatible Possion structures for Lax equations on Possion algebras, Comm. Math. Phys., 203(1999), p.573-592

[30] Y. Nutku and M.V. Pavlov, Multi-Lagrangians for integrable systems, J. Math. Phys., 43(2002), p.1441-p.1459

[31] P.J. OLver, Applications of Lie-Groups to Differential Equations, SpringerVerlag, New York, 1993

[32] P.J. Olver and Y.Nutku, Hamiltonian structures for systems of hyperbolic conservation Laws, J. Math. Phys, 29(1988), p.1610-p.1619

[33] M.V. Pavlov, Classifying integrable hydrodynamic Egoroff chains, Theo. Math. Phys., 138(2004),p.45-p.58

[34] M.B. Sheftel, Group analysis of hydrodynamic-type systems, arXiv:math-th/0104014

[35] J.Sidorenko and W.Strampp, Symmetry constraints of the KP hierarchy, Inverse Problems, 7(1991), L.37-L.43

[36] I.A.B. Strachan, A geometry for multidimensional integrable systems, J. Geom. Phys., 21(1997), p.255-p.278

[37] K. Takasaki and T. Takebe, Integrable hierarchies and dispersionless limit, Rev. Math. Phys., 7(1995),p.743

[38] V.E. Zakharov, Benney equation and quasi-classical approximation in the inverse problem method, Funkts. Anal. Prilozh, 1980, p.89-p.98 